\title{
Identification of Serial DNA Methylation Changes in the Blood Samples of Patients with Lung Cancer
}

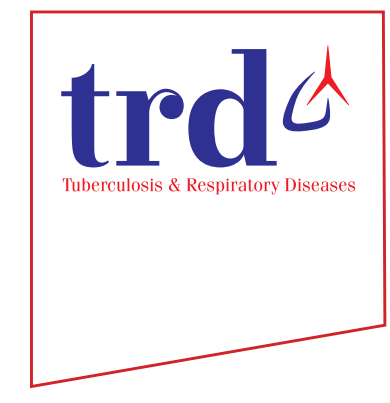

\author{
Da Hye Moon, M.D. ${ }^{1}{ }^{\mathbb{D}}$, Sung Ok Kwon, Ph.D. ${ }^{2}$, Woo Jin Kim, M.D. ${ }^{1,3}$ and Yoonki Hong, M.D. ${ }^{1,3}$ \\ ${ }^{1}$ Department of Internal Medicine, ${ }^{2}$ Biomedical Research Institute, Kangwon National University Hospital, Chuncheon, \\ ${ }^{3}$ Department of Internal Medicine, Kangwon National University School of Medicine, Chuncheon, Korea
}

Background: The development of lung cancer results from the interaction between genetic mutations and dynamic epigenetic alterations, although the exact mechanisms are not completely understood. Changes in DNA methylation may be a promising biomarker for early detection and prognosis of lung cancer. We evaluated the serial changes in genomewide DNA methylation patterns in blood samples of lung cancer patients.

Methods: Blood samples were obtained for three consecutive years from three patients ( 2 years before, 1 year before, and after lung cancer detection) and from three control subjects (without lung cancer). We used the MethylationEPIC BeadChip method, which covers the 850,000 bp cytosine-phosphate-guanine (CpG) site, to conduct an epigenome-wide analysis. Significant differentially methylated regions (DMRs) were identified using p-values $<0.05$ in a correlation test identifying serial methylation changes and serial increase or decrease in $\beta$ value above 0.1 for three consecutive years.

Results: We found three significant CpG sites with differentially methylated $\beta$ values and 7,105 CpG sites with significant correlation from control patients without lung cancer. However, there were no significant DMRs. In contrast, we found 11 significant $\mathrm{CpG}$ sites with differentially methylated $\beta$ values and 10,562 CpG sites with significant correlation from patients with lung cancer. There were two significant DMRs: cg21126229 (RNF212) and cg27098574 (BCAR1).

Conclusion: This study revealed DNA methylation changes that might be implicated in lung cancer development. The DNA methylation changes may be the possible candidate target regions for the early detection and prevention of lung cancer.

Keywords: DNA Methylation; Lung Neoplasms; Biomarkers

\section{Address for correspondence: Yoonki Hong, M.D.}

Department of Internal Medicine, Kangwon National University School of Medicine, 1 Gangwondaehak-gil, Chuncheon 24341, Korea

Phone: 82-32-258-9364, Fax: 82-32-258-2404

E-mail: h-doctor@hanmail.net

*The abstract of this paper was presented at the IASLC 18th World Conference on Lung Cancer, which was held 15-18 Oct 2017 at the Conference Center, Pacifico Yokohama, Japan.

Received: May. 6, 2018

Revised: Jun. 21, 2018

Accepted: Jul. 31, 2018

Published online: Sep. 28, 2018

(c) It is identical to the Creative Commons Attribution Non-Commercial License (http://creativecommons.org/licenses/by-nc/4.0/). The Korean Academy of Tuberculosis and Respiratory Diseases.

\section{Introduction}

Worldwide, lung cancer is the leading cause of cancer death ${ }^{1}$. Although the survival rate trends have increased in recent years (due to early detection by low-dose computed tomography $[\mathrm{CT}]$ screening and new therapeutic agents, such as molecular targeted agents or immunotherapy), the 5-year survival rate remains poor, at about $20 \%-30 \%^{2,3}$. Therefore, in the area of lung cancer research, it is an important task still to identify biomarkers that may be useful for early diagnosis.

The genetic changes found in lung cancer can mainly be explained by the interaction between permanent genetic mutations and dynamic epigenetic changes ${ }^{4}$. Since permanent genetic alterations in lung cancer have been studied extensively there has been substantial progress in molecular-targeted 
therapeutics in the treatment of patients with non-small-cell lung cancer (NSCLC) ${ }^{5}$. However, the overall survival rate reduction effect of the molecular targeted therapeutics for lung cancer is insignificant. Although somatic genetic mutations might play a major role in cancer development, epigenetic changes are known to play more variable roles, since they can either repress the expression of tumor suppressor genes or activate the expression of oncogenes ${ }^{4,6}$. Therefore, epigenetic changes could be a target to uncover biomarkers for the early detection of lung cancer.

Epigenetic changes are defined as heritable modifications in gene expression which do not alter the DNA coding sequence directly ${ }^{7}$. Over the past decade, they have been studied increasingly as biomarkers for the early detection of cancer and therapeutic targets ${ }^{8,9}$. Epigenetic changes observed in oncogenesis consist of aberrant DNA methylation patterns, histone modifications, and regulation by noncoding RNAs ${ }^{7}$. DNA methylation is the most studied epigenetic change and is known to repress gene expression and maintain genomic stability ${ }^{9}$. Therefore, DNA methylation study may give a potential clue for identifying biomarkers for early detection of lung cancer or to predict tumor progression.

We evaluated serial changes in genome-wide DNA methylation patterns in blood samples from lung cancer patients.

\section{Materials and Methods}

\section{Study subjects and the preparation of tissue samples}

For this study, we used blood samples obtained from a Korean chronic obstructive pulmonary disease (COPD) $\operatorname{cohort}^{10}$. They were prospectively recruited from 2012 to 2015 to observe clinical outcomes of Koreans living near cement plants. They will be followed up for 10 years and will be monitored for clinical information, blood/urine samples, and surveyed via questionnaire. The cohort recruited 445 patients from 2012 till
December 2015. Among them, five patients developed lung cancer till the end of 2016, two of small cell lung cancer and three of NSCLC. Among them, blood samples were obtained for three consecutive years from three patients who had developed NSCLC ( 2 years before, 1 year before, and after lung cancer detection) and from three control subjects who did not have lung cancer and were matched for age and smoking history (Figure 1). Appropriate informed consent was obtained from all the patients, and the Institutional Review Board of Kangwon National University Hospital approved the study (IRB no. KNUH2012-06-007). All the COPD cohort study conduct adheres to Good Clinical Practice Guidelines and the tenets of the Declaration of Helsinki.

\section{Genomic DNA preparation and DNA methylation profiling}

We conducted an epigenome-wide analysis using a MethylationEPIC BeadChip kit, which covers the 850,000 bp cytosine-phosphate-guanine $(\mathrm{CpG})$ site. The methylation value $(\beta)$, a ratio between methylated probe intensity and total probe intensity, was interpreted as the proportion of methylation; $\beta$ values range from 0 (unmethylated) to 1 (methylated).

The DNA quality was checked with a spectrophotometer (NanoDrop ND-1000 UV-vis; NanoDrop Technologies, Wilmington, DE, USA), and genomic DNA was diluted to $50 \mathrm{ng} / \mu \mathrm{L}$ using a Quant-iT PicoGreen quantitation assay (Invitrogen, Carlsbad, CA, USA). Bisulfite-conversion using an EZ DNA methylation kit (Zymo Research, Irvine, CA, USA) was carried out according to the manufacturer's protocols (Supplementary Methods).

\section{Statistical analysis}

We used methylation $\beta$ values because they are more easily interpretable as methylation changes than $\mathrm{M}$ values are, the $\log _{2}$ ratio of methylated probe intensity and unmethylated

\begin{tabular}{|c|c|c|}
\hline Sample time & Sample group & Trait \\
\hline \multirow{3}{*}{$\begin{array}{l}\text { Before } \\
\text { two years }\end{array}$} & Patient 1 & \multirow{3}{*}{1} \\
\hline & Patient 2 & \\
\hline & Patient 3 & \\
\hline \multirow{3}{*}{$\begin{array}{l}\text { Before } \\
\text { one year }\end{array}$} & Patient 1 & \multirow{3}{*}{2} \\
\hline & Patient 2 & \\
\hline & Patient 3 & \\
\hline \multirow{3}{*}{$\begin{array}{c}\text { After } \\
\text { lung cancer }\end{array}$} & Patient 1 & \multirow{3}{*}{3} \\
\hline & Patient 2 & \\
\hline & Patient 3 & \\
\hline
\end{tabular}

Delta mean $\&$ t-test
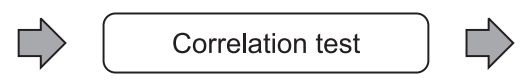

Significant filtering
Figure 1. Blood samples were obtained for three consecutive years from three patients with non-small-cell lung cancer or control subjects and analyzed by delta mean and correlation test. Significant $\beta$ changes were defined as when the delta mean of trait 2-1 and 3-2 was increased $(\geq 0.1)$ or decreased $(\leq-0.1)$, and significant correlation was defined when the $\mathrm{p}$ value for the correlation test was $>0.05$. When both conditions were satisfied, significant differentially-methylated regions were obtained. dm: delta mean. 
probe intensity.

In three patients who had developed lung cancer, we assigned continuous labels to each patient in the order of the sample collection year ( 1,2 years before lung cancer detection; 2, 1 year before lung cancer detection; and 3, after lung cancer detection). The mean differentially-methylated level ( $\beta$ value) of each trait and correlation test, according to continuous sample collection order, was evaluated. Significant $\beta$ changes were defined when the delta mean of trait $2-1$ and $3-2$ was increased or decreased by a factor of at least 0.1 . Significant correlation was defined as when the p-value for the correlation test was $<0.05$. Significant differentially methylated regions were obtained when both conditions were satisfied (Figure 1).

In addition, in the three control subjects, we assigned the continuous labels 1 to 3 in the order of sample collection year ( 1 , first year; 2 , second year; and 3 , third year). The mean differentially methylated level ( $\beta$ values) of each trait and correlation test, according to continuous sample collection order, were evaluated. Significant $\beta$ changes were defined when the delta mean of trait 2-1 and 3-2 was increased or decreased by a factor of at least 0.1 , and significant correlation was defined when the $p$-value for the correlation test was $>0.05$. When both conditions were satisfied, significant differentially methylated regions were obtained.

All the methylation data processing, statistical analyses, and visualizations were conducted in R 3.0.2 (R Foundation for Statistical Computing, Vienna, Austria).

\section{Results}

The baseline characteristics of patients and control were summarized on Table 1.

We identified 11 regions with significant methylation changes in the three patients with lung cancer. The 11 regions were as follows: cg02740582, cg05130642, cg07850982, cg16057201, cg16298462, cg19536810, cg19565594, cg21126229, cg25304620, cg25994476, and cg27098574. In addition, 10,562 regions were identified as having significant correlation for continuous years. Significant differentiallymethylated regions satisfying both conditions were found in two regions: cg21126229 and cg27098574 (Figure 2). The related genes for cg21126229 and cg27098574 were RNF212 and $B C A R 1$. Figure 3 presents the clustering heatmaps.

Three regions with significant methylation changes were identified in the three control subjects. In addition, 7,105 regions were identified as having significant correlation for continuous years. However, there were no significant differentially-methylated regions which satisfied both conditions.

Meanwhile, to observe DNA methylation changes from another perspective, we analyzed cancer and control subjects by grouping them with same sample collection years. We sought to select a position that showed the difference between the cancer and the control samples at the same time, ultimately to show the difference between the cancer samples and the controls, and, at the same time, to show the position indicating the serial change. However, we did not find significant differentially-methylated regions with significant methylation changes and correlation for continuous years.

\section{Discussion}

In this study, we identified differentially methylated regions associated with the development of NSCLC in 3-year serial blood samples obtained from patients with and without NSCLC. Two genes, RNF212 and BCAR1, that were associated with occurrence or early detection of NSCLC were found. Our study results may be used as a resource for finding biomarkers for the early detection of lung cancer.

Lung cancer is clinically manifested in various presentations and has few symptoms at the early stages. Early symptoms are subtle and non-specific and may lead to initial diagnosis being at an advanced stage for many patients with lung cancer. Although low dose CT screening was introduced as a promising strategy for the early detection of lung cancer ${ }^{11,12}$, because it has several limitations, such as the possibility of lead time bias, a high rate of false-positive detection, and the as yet unanswered questions of radiation hazard effects, the debate continues ${ }^{13}$. The early detection of lung cancer may be more urgently needed. Epigenetic biomarkers such as DNA methylation and non-coding RNA may become alternative diagnostic methods to detect lung cancer in its earlier stages. The incidence of aberrant DNA methylation in cancer is higher than that of somatic mutation, and, since global DNA demethylation has been found in most lung cancers, it has the possibility to be a diagnostic marker for lung cancer ${ }^{4,9}$. Recent studies have suggested that gene-specific promoter DNA

Table 1. Baseline characteristics of patients with lung cancer and matched control subjects

\begin{tabular}{|c|c|c|}
\hline & Patient $(n=3)$ & Control $(n=3)$ \\
\hline Age, yr & $69(60-74)$ & $66(57-68)$ \\
\hline Smoking pack year, yr & $20(3-50)$ & $20(0.2-50)$ \\
\hline Post bronchodilator $\mathrm{FEV}_{1}$ & $92(46-106)$ & $76(76-77)$ \\
\hline \multicolumn{3}{|l|}{ Histological type } \\
\hline Adenocarcinoma & $1(33)$ & - \\
\hline Squamous cell carcinoma & $2(67)$ & - \\
\hline Stage II & $2(67)$ & - \\
\hline Stage III & $1(33)$ & - \\
\hline
\end{tabular}

Values are presented as median (range) or number (\%).

$\mathrm{FEV}_{1}$ : forced expiratory volume on one second. 


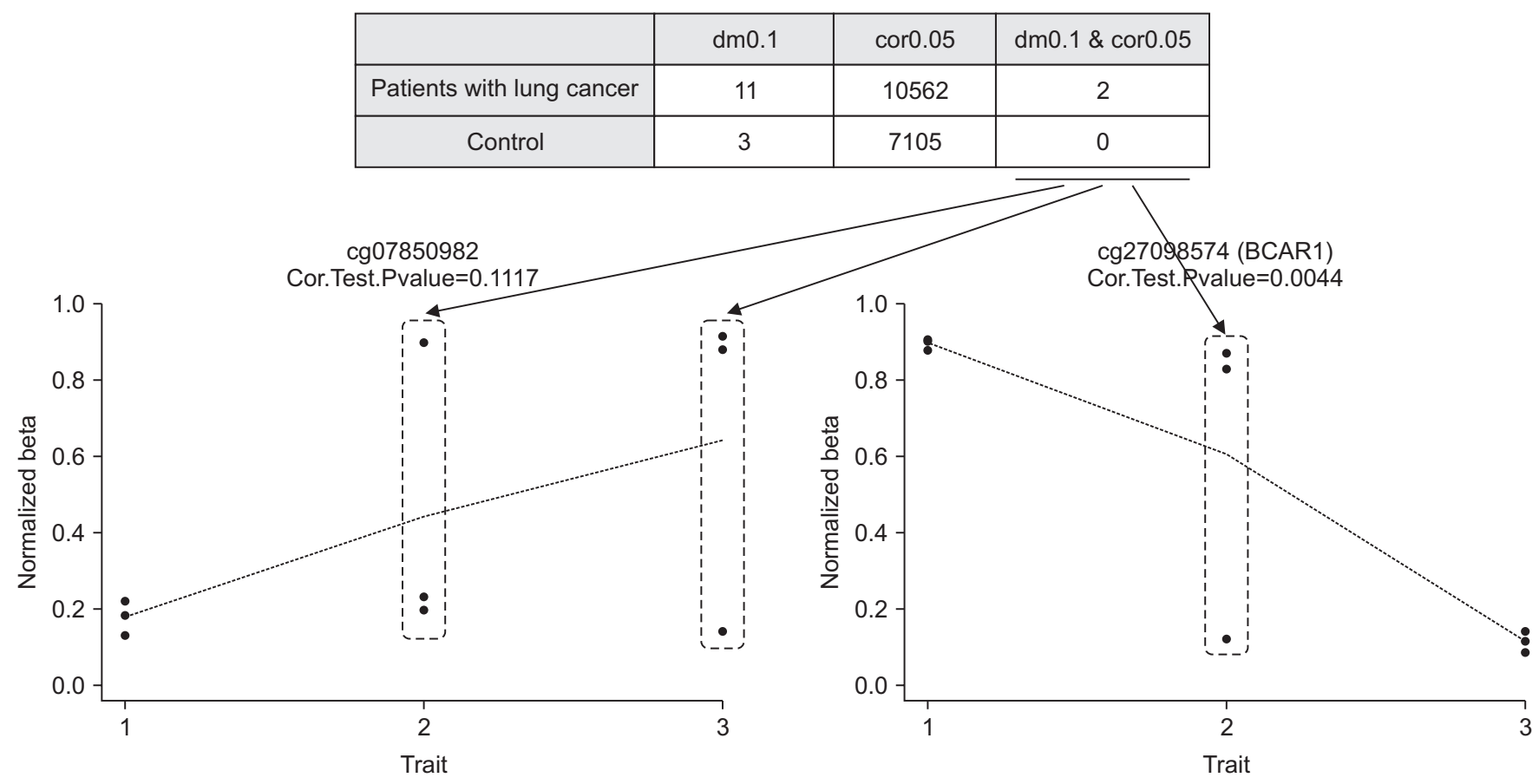

Figure 2. Significant differentially methylated regions with significant $\beta$ changes and correlation. dm0.1: significant $\beta$ changes of the delta mean with increased $(\geq 0.1)$ or decreased $(\leq-0.1)$; cor 0.05 : significant correlation with p-value of $>0.05$.

methylation may be a useful biomarker for the early detection of lung cancer or for the prediction of lung cancer outcomes ${ }^{4,9}$.

The aberrant promoter methylation changes in NSCLC have been identified in several genes from various samples. RASSF1A in sputum ${ }^{14}, S H O X 2$ in bronchial aspirates ${ }^{15}$, and CDKN2A in bronchoalveolar lavage and exhaled breath condensates ${ }^{16,17}$ have been found. In tumor tissues, Brock et al. ${ }^{18}$ showed that tumor recurrence was associated with differentially methylated regions of four gene promoters ( $p 16$, CDH13, APC, and RASSF1A); and Sandoval et al. ${ }^{19}$ identified hypermethylations of genes (HISTIH4F, PCDHGB6, NPBWR1, ALX1, and HOXA9) were associated with shorter survival. These studies have shown the possibility that DNA methylation biomarkers might improve diagnostic accessibility or prognosis prediction beyond standard staging. However, biomarkers for the early detection of lung cancer are in development only and it is expected that they are unlikely to be clinically applied within the next few years ${ }^{8}$. For a biomarker in clinical practice, it may be needed to be detected easily and early in blood. Promotor hypermethylation of six genes (RASSF1A, CDKN2A, RARb, CDH13, FHIT, and BLU) showed approximately $80 \%$ concordance between serum and tissue samples in NSCLC ${ }^{20}$. However, promoter methylation of $C D K N 2 A, D A P K, P A X 5 b$, and GATA5 in blood was much lower than in tissue samples ${ }^{21}$. These studies show that DNA promotor methylation in blood already has limitations for the early detection of lung cancer, because it assumes that DNA methylation changes in serum or plasma were found in DNA from apoptotic tumor cells, reflecting an advanced stage. Therefore, using an epigenome-wide associated study to identify new methylation changes of normal cells mediated by cancer cells, as immune cells, may open a new pathway for finding a biomarker for the early detection of lung cancer.

To understand DNA methylation changes induced by cancer, methylation across the whole genome should be measured simultaneously. However, whole-genome bisulphite sequencing may not be the most appropriate method because of its high cost and the technical expertise required to conduct it although it is considered a gold standard tool ${ }^{22}$. However, a microarray might be used as a popular and informative alternative. The Illumina Infinium BeadChips, the HumanMethylation27K and $450 \mathrm{~K}$ BeadChip, have been introduced as tools for genome-wide DNA methylation profiling and have been affirmed as having easy and effective characteristics ${ }^{23}$. Recently, a more advanced tool containing over 850,000 probes, the Infinium MethylationEPIC (EPIC) BeadChip, with an increased genome coverage of regulatory regions, was introduced. With high reproducibility and reliability, the EPIC array is a significant improvement over the $27 \mathrm{~K}$ and $450 \mathrm{~K}$ microarrays $^{24}$. Therefore, it may allow new valuable insights into the role of DNA methylation changes in cancer. This study, to our knowledge, is the first that uses the EPIC array to show DNA methylation changes in the blood samples of lung cancer patients.

Our study showed two genes associated with DNA methylation changes in lung cancer, RNF212 and BCRA1. Stud- 


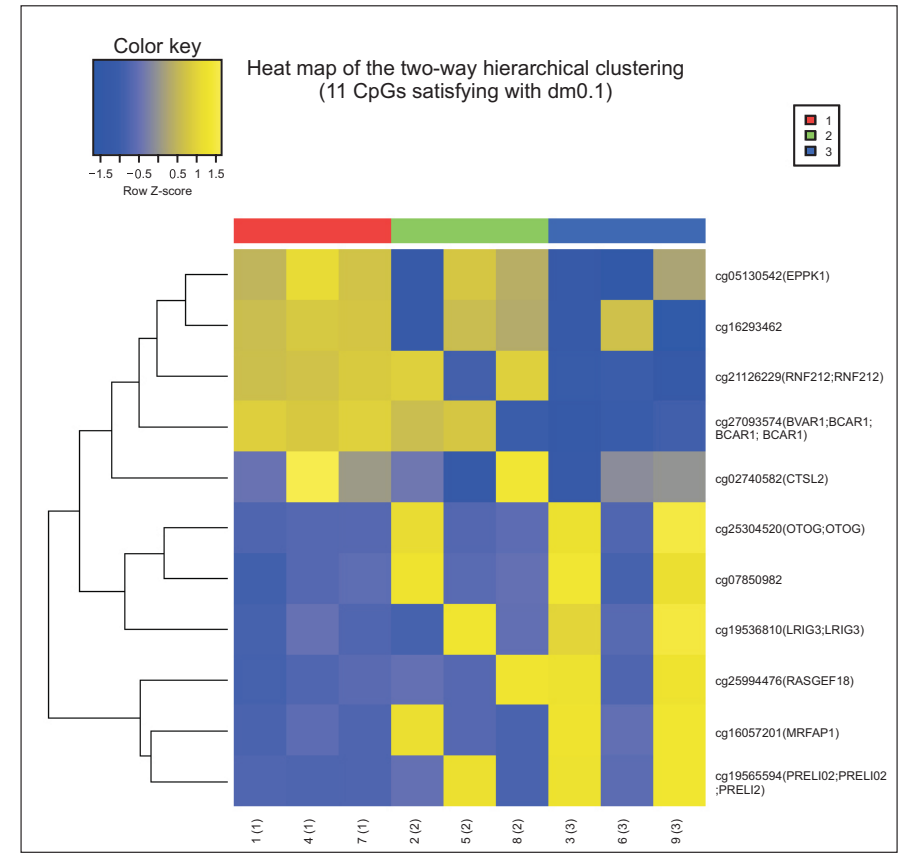

$\mathrm{dm} 0.1$

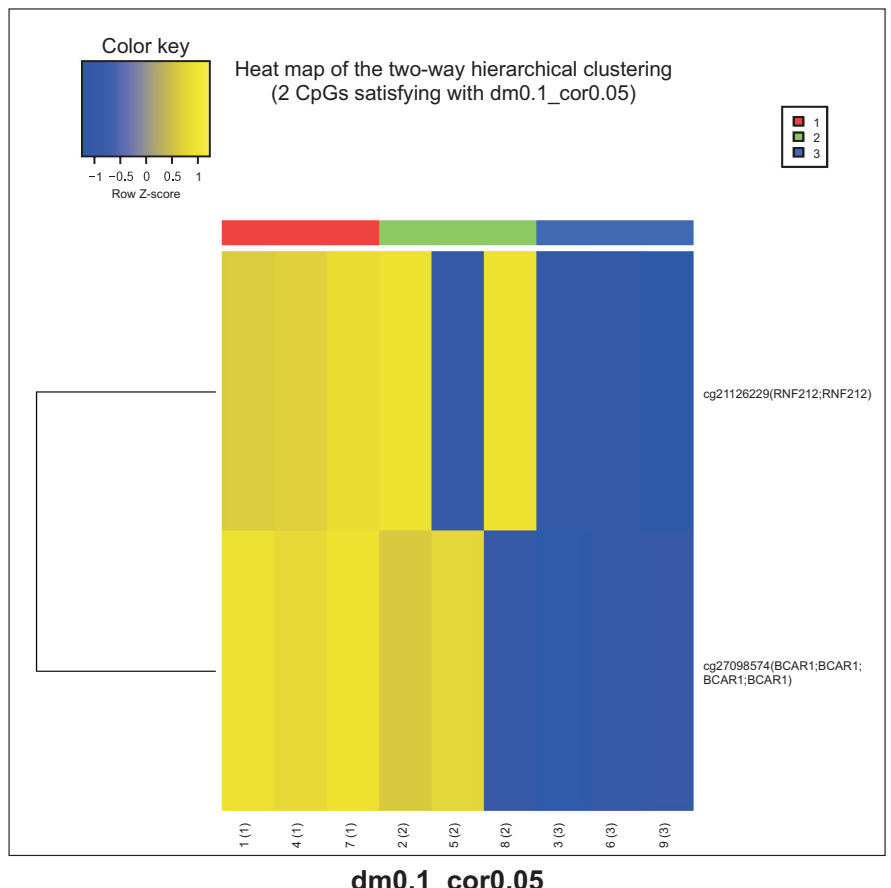

dm0.1_cor0.05

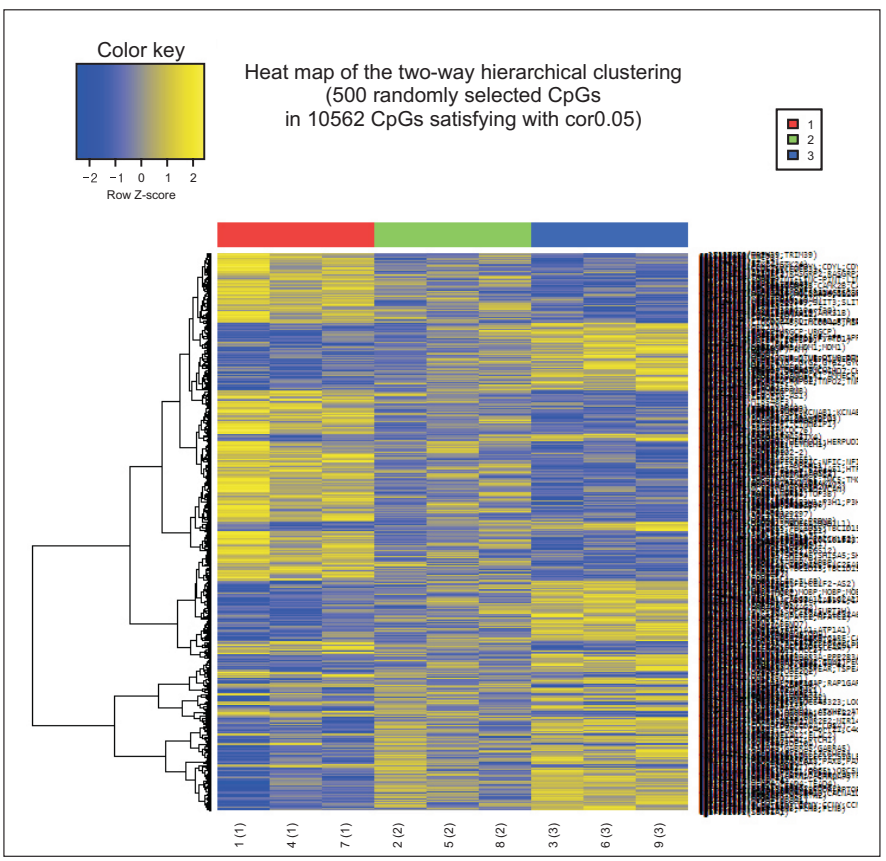

cor0.05

Figure 3. Clustering heatmap of three patients with lung cancer. $\operatorname{dm} 0.1$ : significant $\beta$ changes of the delta mean with increased $(\geq 0.1)$ or decreased ( $\leq-0.1)$; cor0.05: significant correlation with p-value of $>0.05$.

ies have reported that the $R N F 212$ gene is associated with variation in the genome-side recombination rate and acts to stabilize meiosis-specific recombination factors ${ }^{25,26}$. There has been no reported association between $R N F 212$ and lung cancer. Further research is needed. Meanwhile, $B C R A$ is a wellknown gene whose role in lung cancer has been well studied. The BRCA gene acts as a tumor suppressor; its germ line mutation increases the risk of breast and ovarian cancer ${ }^{27}$. In

early stage NSCLC, the DNA methylation of $B R C A 1$ and high mRNA expression of BRCA 1 is associated with poorer surviv$\mathrm{al}^{28,29}$. However, to date, there is low-level evidence to support the use of $B R C A$ as a clinically relevant biomarker in NSCLC. More precise and specific mechanism studies are needed.

This genome-wide DNA methylation study showed DNA methylation changes that might be implicated in the development of lung cancer. The DNA methylation changes may be 
candidate target regions for the early detection and prevention of lung cancer. Further investigation of these DNA methylation changes and related genes may enhance development of a biomarker and/or clinical applications for lung cancer.

\section{Authors' Contributions}

Conceptualization: Kim WJ, Hong Y. Methodology: Moon DH, Kim WJ, Hong Y. Formal analysis: Kwon SO. Kim WJ, Hong Y. Data curation: Kwon SO, Software: Kwon SO. Validation: Kwon SO, Kim WJ, Hong Y. Investigation: Moon DH, Kim WJ, Hong Y. Writing -original draft preparation: Moon DH, Hong Y. Writing - review and editing: all authors. Approval of final manuscript: all authors.

\section{Conflicts of Interest}

No potential conflict of interest relevant to this article was reported.

\section{Acknowledgments}

This study was supported by a grant from the National R\&D Program for Cancer Control, Ministry for Health and Welfare, Republic of Korea (1631210).

\section{Supplementary Material}

Supplementary material can be found in the journal homepage (http://www.e-trd.org).

Supplementary Methods. Genomic DNA preparation and DNA methylation profiling.

\section{References}

1. Global Burden of Disease Cancer Collaboration, Fitzmaurice C, Dicker D, Pain A, Hamavid H, Moradi-Lakeh M, et al. The global burden of cancer 2013. JAMA Oncol 2015;1:505-27.

2. Park JY, Jang SH. Epidemiology of lung cancer in Korea: recent trends. Tuberc Respir Dis 2016;79:58-69.

3. Shin A, Oh CM, Kim BW, Woo H, Won YJ, Lee JS. Lung cancer epidemiology in Korea. Cancer Res Treat 2017;49:616-26.

4. Balgkouranidou I, Liloglou T, Lianidou ES. Lung cancer epigenetics: emerging biomarkers. Biomark Med 2013;7:49-58.

5. Devarakonda S, Morgensztern D, Govindan R. Genomic alterations in lung adenocarcinoma. Lancet Oncol 2015;16:e34251.

6. Esteller M. Non-coding RNAs in human disease. Nat Rev
Genet 2011;12:861-74.

7. Zhang Y. Recent progress in the epigenetics and chromatin field. Cell Res 2011;21:373-4.

8. Ansari J, Shackelford RE, El-Osta H. Epigenetics in non-small cell lung cancer: from basics to therapeutics. Transl Lung Cancer Res 2016;5:155-71.

9. Mehta A, Dobersch S, Romero-Olmedo AJ, Barreto G. Epigenetics in lung cancer diagnosis and therapy. Cancer Metastasis Rev 2015;34:229-41.

10. Hong Y, Ji W, An S, Han SS, Lee SJ, Kim WJ. Sex differences of COPD phenotypes in nonsmoking patients. Int J Chron Obstruct Pulmon Dis 2016;11:1657-62.

11. Oken MM, Hocking WG, Kvale PA, Andriole GL, Buys SS, Church TR, et al. Screening by chest radiograph and lung cancer mortality: the Prostate, Lung, Colorectal, and Ovarian (PLCO) randomized trial. JAMA 2011;306:1865-73.

12. National Lung Screening Trial Research Team, Aberle DR, Adams AM, Berg CD, Black WC, Clapp JD, et al. Reduced lung-cancer mortality with low-dose computed tomographic screening. N Engl J Med 2011;365:395-409.

13. van der Aalst CM, Ten Haaf K, de Koning HJ. Lung cancer screening: latest developments and unanswered questions. Lancet Respir Med 2016;4:749-61.

14. Hubers AJ, Brinkman P, Boksem RJ, Rhodius RJ, Witte BI, Zwinderman $\mathrm{AH}$, et al. Combined sputum hypermethylation and eNose analysis for lung cancer diagnosis. J Clin Pathol 2014;67:707-11.

15. Kneip C, Schmidt B, Seegebarth A, Weickmann S, Fleischhacker M, Liebenberg V, et al. SHOX2 DNA methylation is a biomarker for the diagnosis of lung cancer in plasma. J Thorac Oncol 2011;6:1632-8.

16. Ahrendt SA, Chow JT, Xu LH, Yang SC, Eisenberger CF, Esteller M, et al. Molecular detection of tumor cells in bronchoalveolar lavage fluid from patients with early stage lung cancer. J Natl Cancer Inst 1999;91:332-9.

17. Xiao P, Chen JR, Zhou F, Lu CX, Yang Q, Tao GH, et al. Methylation of P16 in exhaled breath condensate for diagnosis of non-small cell lung cancer. Lung Cancer 2014;83:56-60.

18. Brock MV, Hooker CM, Ota-Machida E, Han Y, Guo M, Ames S, et al. DNA methylation markers and early recurrence in stage I lung cancer. N Engl J Med 2008;358:1118-28.

19. Sandoval J, Mendez-Gonzalez J, Nadal E, Chen G, Carmona FJ, Sayols S, et al. A prognostic DNA methylation signature for stage I non-small-cell lung cancer. J Clin Oncol 2013;31:41407.

20. Hsu HS, Chen TP, Hung CH, Wen CK, Lin RK, Lee HC, et al. Characterization of a multiple epigenetic marker panel for lung cancer detection and risk assessment in plasma. Cancer 2007;110:2019-26.

21. Belinsky SA, Grimes MJ, Casas E, Stidley CA, Franklin WA, Bocklage TJ, et al. Predicting gene promoter methylation in non-small-cell lung cancer by evaluating sputum and serum. Br J Cancer 2007;96:1278-83. 
22. Stirzaker C, Taberlay PC, Statham AL, Clark SJ. Mining cancer methylomes: prospects and challenges. Trends Genet 2014;30:75-84.

23. Bibikova M, Le J, Barnes B, Saedinia-Melnyk S, Zhou L, Shen $\mathrm{R}$, et al. Genome-wide DNA methylation profiling using Infinium(R) assay. Epigenomics 2009;1:177-200.

24. Pidsley R, Zotenko E, Peters TJ, Lawrence MG, Risbridger GP, Molloy P, et al. Critical evaluation of the Illumina MethylationEPIC BeadChip microarray for whole-genome DNA methylation profiling. Genome Biol 2016;17:208.

25. Qiao H, Prasada Rao HB, Yang Y, Fong JH, Cloutier JM, Deacon DC, et al. Antagonistic roles of ubiquitin ligase HEI10 and SUMO ligase RNF212 regulate meiotic recombination. Nat
Genet 2014;46:194-9.

26. Sandor C, Li W, Coppieters W, Druet T, Charlier C, Georges M. Genetic variants in REC8, RNF212, and PRDM9 influence male recombination in cattle. PLoS Genet 2012;8:e1002854.

27. Foulkes WD. Inherited susceptibility to common cancers. N Engl J Med 2008;359:2143-53.

28. Marsit CJ, Liu M, Nelson HH, Posner M, Suzuki M, Kelsey KT. Inactivation of the Fanconi anemia/BRCA pathway in lung and oral cancers: implications for treatment and survival. Oncogene 2004;23:1000-4.

29. Rosell R, Skrzypski M, Jassem E, Taron M, Bartolucci R, Sanchez JJ, et al. BRCA1: a novel prognostic factor in resected non-small-cell lung cancer. PLoS One 2007;2:e1129. 


\section{Supplementary Methods}

\author{
Genomic DNA preparation and DNA methylation \\ profiling
}

\section{Genomic DNA quantitation}

DNA samples were checked the quality using NanoDrop ND-1000 UV-Vis spectrophotometer (Wilmington, DE, USA). Then samples were electrophoresed on agarose gels and samples with intact genomic DNA showing no smearing on agarose gel electrophoresis were selected for experiment. Intact genomic DNA was diluted to $50 \mathrm{ng} / \mu \mathrm{L}$ concentration based on Quant-iT Picogreen (Invitrogen, Carlsbad, CA, USA) quantitation. Concentrations were adjusted based on these results. All prepared samples were bisulfite-converted according to the Zymo EZ DNA methylation kit protocols (Zymo Research, Orange, CA, USA).

\section{Bisulfite conversion (Zymo EZ DNA methylation kit)}

Five hundred nanograms of input gDNA was required for the bisulfite conversion. Add conversion reagent and incubate in a thermocycler to denature. CT converted DNA was washing and desulphonating with desulphonation buffer. After desulphonation, the DNA was washing again and eluting with $12 \mu$ L elution buffer.

\section{Sample amplification and hybridization for Bead- Chips (MethylationEPIC DNA Analysis BeadChip Kit; Illumina, San Diego, CA, USA)}

The whole-genome amplification process requires $200 \mathrm{ng}$ of input bisulfite-converted DNA, MAl and creates a sufficient quantity of DNA ( $1,000 \times$ amplification) to be used on a single BeadChip in the infinium methylation assay (Illumina RPM and MSM). After amplification, the product is fragmented using a proprietary reagent (FMS), precipitated with 2-propanol (plus precipitating reagent; $\mathrm{PM1}$ ), and resuspended in formamide-containing hybridization buffer (RAl). The DNA samples are denatured at $95^{\circ} \mathrm{C}$ for 20 minutes, then placed in a humidified container for a minimum of 16 hours at $48^{\circ} \mathrm{C}$ allowing CpG loci to hybridize to the 50mer capture probes.

\section{Allele specific single-base extension and staining on BeadChips}

Following hybridization, the BeadChip/Te-Flow chamber assembly was placed on the temperature-controlled Tecan Flowthrough Chamber Rack, and all subsequent washing, extension, and staining were performed by addition of reagents to the Te-Flow chamber.

For the allele specific single-base extension assay, primers were extended with a polymerase and labeled nucleotide mix (TEM), and stained with repeated application of STM (staining reagent) and ATM (anti-staining reagent). After staining was complete, the slides were washed with low salt wash buffer
(PB1), immediately coated with XC4, and then imaged on the Illumina iScan Reader.

\section{Imaging the BeadChip and data analysis}

The Illumina iScan Reader is a two-color $(532 \mathrm{~nm} / 658 \mathrm{~nm})$ confocal fluorescent scanner with $0.53-\mu \mathrm{m}$ pixel resolution. The scanner excites the fluorophors generated during signal amplification/staining of the allele-specific (one color) extension products on the BeadChips. The image intensities are extracted using Illumina's iScan Control software.

\section{Raw data preparation and Statistical analysis}

The quality of hybridization and overall chip performance were monitored by visual inspection of both internal quality control checks and the raw scanned data. Raw data were extracted as beta values for each $\mathrm{CpG}$ for each sample using $\mathrm{R}$ watermelon package. Beta values were calculated by subtracting background using negative controls on the array and taking the ratio of the methylated signal intensity against the sum of both methylated and unmethylated signals. A beta value of 1-1.0 was reported significant percent methylation, from $0 \%$ to $100 \%$, respectively, for each CpG site.

Array CpG probes that have detection p-value $\geq 0.05$ (similar to signal to noise) in over $25 \%$ samples were filtered out. (We applied a filtering criterion for data analysis; good signal value was required to obtain a detection $\mathrm{p}$-value $<0.05$ ). And then filtered data was background correction \& dye bias equalization by R methylumi \& lumi package. To reduce Infinium I and Infinium II assay bias, corrected signal value was normalized by BMIQ (Beta Mixture Quantile) method.

Differentially expressed methylation list was determined using |delta_mean| $\geq 0.2$ (the difference of methylation signal, avg beta of Case-avg beta of Control) and p-value $<0.05$ of independent t-test in which the null hypothesis was that no difference exists among two groups.

All data analysis and visualization of differentially expressed genes was conducted using R 3.0.2 (http://www.r-project.org).

\section{References}

1. Takai D, Jones PA. Comprehensive analysis of CpG islands in human chromosomes 21 and 22. Proc Natl Acad Sci U S A 2002;99:3740-5.

2. Irizarry RA, Ladd-Acosta C, Carvalho B, Wu H, Brandenburg SA, Jeddeloh JA, et al. Comprehensive high-throughput arrays for relative methylation (CHARM). Genome Res 2008;18:78090.

3. Bjornsson HT, Brown LJ, Fallin MD, Rongione MA, Bibikova M, Wickham E, et al. Epigenetic specificity of loss of imprinting of the IGF2 gene in Wilms tumors. J Natl Cancer Inst 2007;99:1270-3.

4. Bibikova M, Lin Z, Zhou L, Chudin E, Garcia EW, Wu B, et al. 
High-throughput DNA methylation profiling using universal bead arrays. Genome Res 2006;16:383-93.

5. Sandoval J, Heyn H, Moran S, Serra-Musach J, Pujana MA,
Bibikova M, et al. Validation of a DNA methylation microarray for $450,000 \mathrm{CpG}$ sites in the human genome. Epigenetics 2011;6:692-702. 